\title{
Cerebral proton magnetic resonance spectroscopy of a patient with giant axonal neuropathy
}

\author{
Knut Brockmann ${ }^{a, *}$, Petra J.W. Pouwels ${ }^{b, 1}$, Peter Dechent ${ }^{b}$, Kevin M. Flanigan ${ }^{c}$, \\ Jens Frahm ${ }^{\mathrm{b}}$, Folker Hanefeld ${ }^{\mathrm{a}}$ \\ ${ }^{a}$ Department of Pediatrics and Neuropediatrics, Georg-August-Universität, Children's Hospital, Robert-Koch-Strasse 40, 37075 Goettingen, Germany \\ ${ }^{\mathrm{b}}$ Biomedizinische NMR Forschungs GmbH am Max-Planck-Institut für Biophysikalische Chemie, Goettingen, Germany \\ ${ }^{c}$ Departments of Neurology, Pathology, and Human Genetics, University of Utah School of Medicine, Salt Lake City, UT, USA
}

Received 26 April 2002; received in revised form 26 July 2002; accepted 14 August 2002

\begin{abstract}
Magnetic resonance imaging of a girl with giant axonal neuropathy revealed a progressive white matter disease. In close agreement with histopathological features reported previously, localized proton magnetic resonance spectroscopy at 9 and 12 years of age indicated a specific damage or loss of axons (reduced $\mathrm{N}$-acetylaspartate and $\mathrm{N}$-acetylaspartylglutamate) accompanied by acute demyelination (elevated cholinecontaining compounds, myo-inositol, and lactate) in white matter as well as a generalized proliferation of glial cells (elevated cholinecontaining compounds and myo-inositol) in both gray and white matter.
\end{abstract}

(C) 2002 Elsevier Science B.V. All rights reserved.

Keywords: Magnetic resonance spectroscopy; Giant axonal neuropathy; Demyelination; Leukoencephalopathy

\section{Introduction}

Amongst the peripheral neuropathies in childhood, giant axonal neuropathy (GAN) shows some unique clinical and morphological features. GAN is a generalized disorder of cytoplasmatic intermediate filaments with accumulation of ovoid aggregates in different cell types [1-3]. Early observations $[4,5]$ depicted clinical features of a peripheral neuropathy in children with 'remarkably kinky hair' [4]. Subsequent reports stressed the involvement of the central nervous system (CNS) as revealed by clinical features [6] as well as neuroradiological [7,8], neurophysiological [9], and neuropathological investigations $[10,11]$. The gene for GAN was mapped to chromosome 16q24 [12]. Recently, the gene GAN has been identified and found to encode for a novel, ubiquitously expressed protein named gigaxonin [13].

Here, we used localized proton magnetic resonance spectroscopy (MRS) to further characterize the involvement of the CNS by assessing metabolic alterations in different brain regions in a girl with GAN at the age of 9 years and in a follow-up investigation three years later.

\footnotetext{
* Corresponding author. Tel.: +49-551-39-6210; fax: +49-551-39-6252. E-mail address: kbrock@med.uni-goettingen.de (K. Brockmann).

${ }^{1}$ Present address: Department of Clinical Physics and Informatics, University Hospital, Vrije Universiteit, Amsterdam, The Netherlands.
}

\section{Clinical report}

The clinical, neurophysiological, and histopathological features of this patient have been described previously [14]. Briefly, muscular hypotonia was noticed at 6 months of age. Her motor development was slightly delayed, she sat unsupported at 15 months, and walked freely at 2 years. She showed a broad-based, atactic gait and developed distal weakness and wasting in her lower limbs. Between the third and fourth year her gait deteriorated and she lost independent walking, standing, and crawling successively over the years. At the age of 4 years clinical and neurophysiological investigations pointed to an axonal polyneuropathy. A sural nerve biopsy was performed and the neuropathological diagnosis of GAN was established.

On re-examination at the age of 9 years, a slowly progressive decline of her psychomotor abilities was evident. She showed muscular hypotonia, marked weakness of arms and legs and distal wasting of lower limbs. By that time she was wheelchair dependent, hardly able to lift her arms and unable to lift her legs against gravity. Plantar reflexes as well as tendon reflexes in arms and legs were absent. There were mild contractures of her knees and severe kyphoscoliosis. Facial weakness, bilateral ptosis, and a 
$9 \mathrm{Y} 8 \mathrm{M}$

12 Y $8 \mathrm{M}$

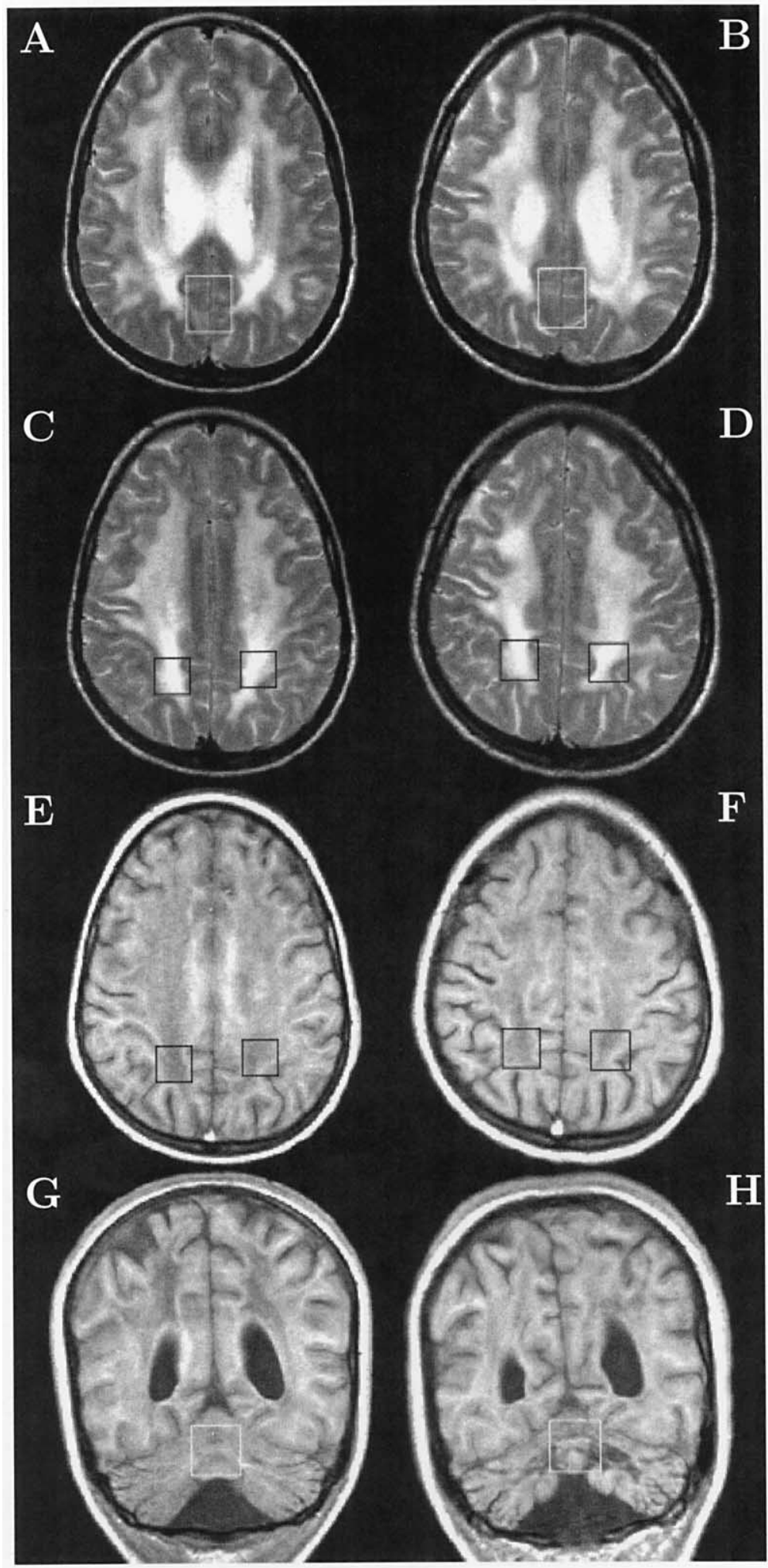

Fig. 1. White matter abnormalities in (A-D) T2-weighted MRI (FSE, TR/TE $=2.625 / 98 \mathrm{~ms}$, flip angles $\left.=90^{\circ} / 120^{\circ}\right)$ and $(\mathrm{E}-\mathrm{H}) \mathrm{T} 1$-weighted MRI $(3 \mathrm{D}$ FLASH, TR/TE $=15 / 4 \mathrm{~ms}$, flip angle $=20^{\circ}$ ) of the patient with giant axonal neuropathy at the age of 9 years (left column) and 12 years (right column). The boxes indicate standardized volumes of interest for proton MRS of paramedian parietal gray matter ( $12.5 \mathrm{ml}, \mathrm{A}, \mathrm{B})$, parieto-occipital white matter (4.1 ml, CF), and central cerebellum ( $8.0 \mathrm{ml}, \mathrm{G}, \mathrm{H})$. 

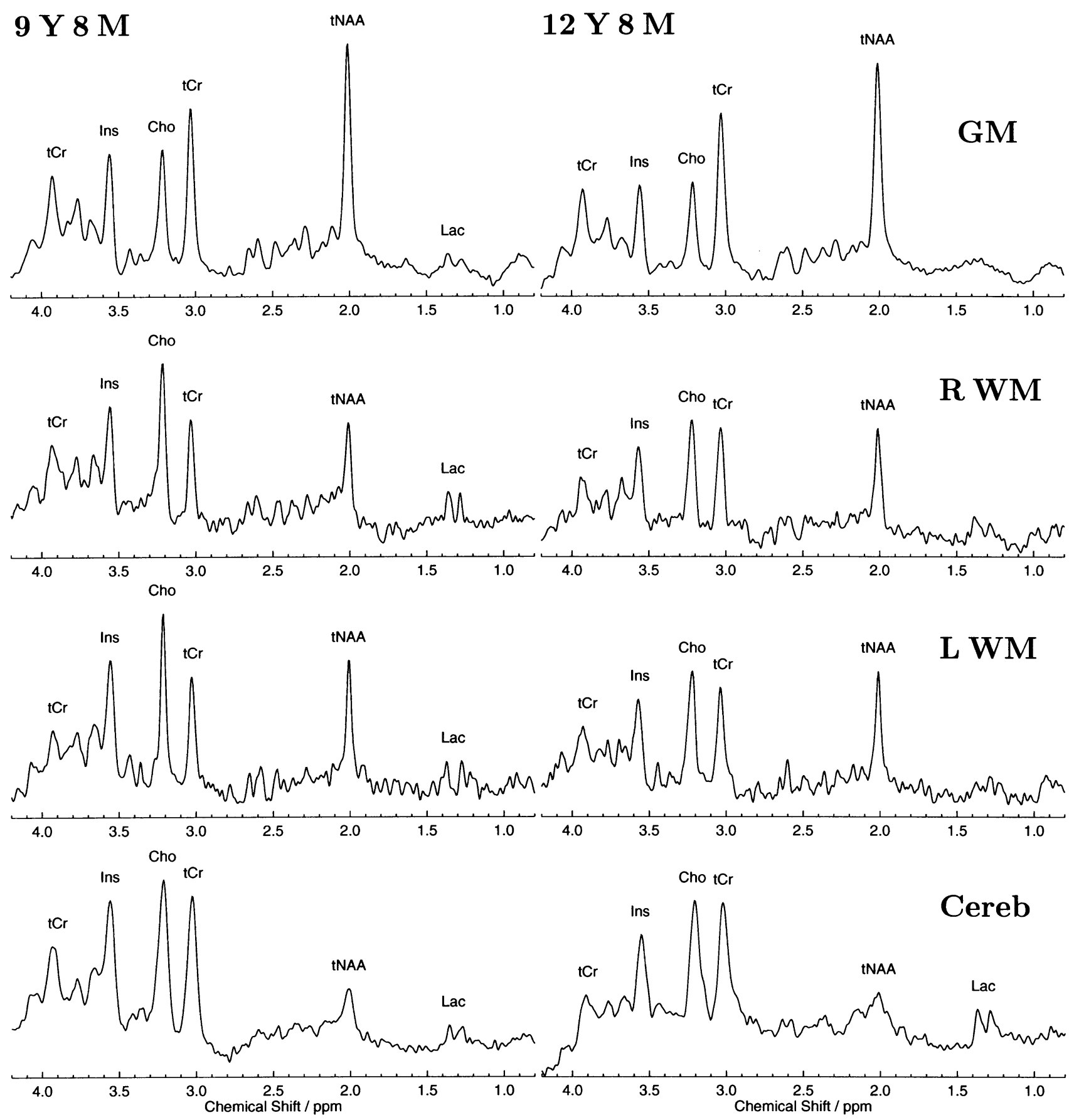

Fig. 2. Proton MRS (TR/TE/TM = 6000/20/10 ms, 64 accumulations) of gray matter (GM), right (R) and left (L) white matter (WM), and cerebellum (Cereb) of the patient with giant axonal neuropathy at the age of 9 years (left column) and 12 years (right column). Major metabolite resonances refer to $N$ acetylaspartate and $\mathrm{N}$-acetylaspartylglutamate (tNAA), creatine and phosphocreatine (tCr), choline-containing compounds (Cho), myo-inositol (Ins), and lactate $(\mathrm{Lac})$.

mild gaze-dependent vertical nystagmus were observed. Optic discs were pale, and visual acuity was diminished.

During the following 3 years she became increasingly incapacitated by weakness and ataxia. At the age of 12 years, she had great difficulties in moving her wheelchair and with control of her head. Dysarthria and dysphagia were apparent. She was attentive and understanding well, though delayed in her response.
Neurophysiological investigations were performed repeatedly during the course of the disease. Nerve conduction studies revealed reduction of amplitudes or absence of compound muscle action potentials, absence of nearly all sensory responses, and mild to moderately reduced conduction velocities. Visual evoked responses were increasingly delayed. Auditory brainstem responses were delayed and finally absent at the age of 11 years. Median nerve somato- 
Table 1

Cerebral metabolite concentrations $(\mathrm{mM})$ of the patient with GAN and of age-matched controls (mean $\pm \mathrm{SD})^{\mathrm{a}}[17]$

\begin{tabular}{|c|c|c|c|c|c|}
\hline & tNAA & $\mathrm{tCr}$ & Cho & Ins & Lac \\
\hline \multicolumn{6}{|l|}{ Gray matter } \\
\hline $9 y 8 m$ & 8.7 & $7.6^{*}$ & $1.9 * *$ & $7.7 * *$ & 1.6 \\
\hline $12 \mathrm{y} 8 \mathrm{~m}$ & 9.0 & $8.1^{* *}$ & $1.5 *$ & $5.8^{*}$ & - \\
\hline Control $(n=22)$ & $9.0 \pm 0.8$ & $6.5 \pm 0.5$ & $1.1 \pm 0.2$ & $4.3 \pm 0.6$ & - \\
\hline \multicolumn{6}{|l|}{ White matter } \\
\hline $9 y 8 \mathrm{~m}$ RPO & $4.1 * *$ & 4.7 & $2.4 * *$ & $6.9^{*}$ & 3.0 \\
\hline 9y 8m LPO & $4.7 * *$ & 5.0 & $2.6 * *$ & $9.1 * *$ & 2.0 \\
\hline $12 \mathrm{y} 8 \mathrm{~m}$ RPO & $4.5 * *$ & 4.8 & 1.9 & $5.7 *$ & - \\
\hline $12 \mathrm{y} 8 \mathrm{~m}$ LPO & $4.6^{* *}$ & 5.2 & $2.1 *$ & $6.0 *$ & - \\
\hline Control $(n=12)$ & $7.9 \pm 0.6$ & $4.8 \pm 0.4$ & $1.6 \pm 0.2$ & $3.9 \pm 1.2$ & - \\
\hline \multicolumn{6}{|l|}{ Cerebellum } \\
\hline $9 y 8 m$ & $3.0 * *$ & $10.1^{*}$ & $3.6 * *$ & $12.0 * *$ & 2.6 \\
\hline $12 \mathrm{y} 8 \mathrm{~m}$ & $2.3 * *$ & 7.4 & $2.9 * *$ & $7.2 *$ & 4.1 \\
\hline Control $(n=3)$ & $7.5 \pm 1.0$ & $8.6 \pm 0.7$ & $2.0 \pm 0.2$ & $4.8 \pm 0.9$ & - \\
\hline
\end{tabular}

a $y$, years; m, months; RPO, right parieto-occipital; LPO, left parietooccipital. $*>2$ SD from control; $* *>4$ SD from control.

sensory evoked responses (SER) were delayed, tibial nerve SER were absent early in the course.

Mutation analysis of the gigaxonin gene was performed by direct sequencing of the entire coding region of the GAN gene. This revealed a homozygous point mutation at $n t 281$ of the consensus sequence (A281G), predicting a codon change from Gln to Arg at residue 94 (Q94A). However, this mutation results in a AG/gt to GG/gt change at the intron 3 donor splice site, and may result in an abnormally spliced transcript.

\section{Methods}

Written informed consent was obtained from the parents. Fully relaxed short-echo time proton MR spectra were acquired with use of a single-voxel stimulated echo acquisition mode localization sequence [15] (TR/TE/TM $=6000 / 20 / 10 \mathrm{~ms}, 64$ accumulations) and the standard imaging headcoil at $2.0 \mathrm{~T}$ (Siemens Magnetom Vision, Erlangen, Germany). Volumes of interest (VOI) were selected from T1-weighted (3D FLASH) and T2weighted (FSE) images, with sizes ranging from 4.1 to $12.5 \mathrm{ml}$ (Fig. 1). Spectral evaluation and quantification of absolute metabolite concentrations were accomplished with use of LCModel, a user-independent fitting routine based on a library of calibrated model spectra of all individual compounds. Details have been described elsewhere [16]. Previous studies of regional age dependencies of cerebral metabolites [17] provided age-matched controls.

Major detectable metabolites include the neuroaxonal markers [18] $N$-acetylaspartate and $N$-acetylaspartylglutamate (tNAA), creatine and phosphocreatine $(\mathrm{tCr})$ as ubiquitous compounds linked to energy metabolism, cholinecontaining compounds (Cho) involved in membrane turn- over, the glial (astrocytic) marker [19] and brain osmolyte myo-inositol (Ins), and lactate (Lac) as the intermediate product of nonoxidative glucose consumption. More specifically, Cho is highly concentrated in oligodendrocytes as indicated by its severe reduction in proton MRS of hypomyelination such as Pelizaeus-Merzbacher disease [20]. It mainly represents contributions from phosphorylcholine and phosphorylethanolamine as precursor molecules for membrane synthesis as well as glycerophosphorylcholine and glycerophosphorylethanolamine as the corresponding membrane degradation products. Elevated Cho and Ins concentrations therefore may indicate both glial proliferation (e.g. in brain tumors), and cellular disruption (e.g. in demyelination), respectively.

\section{Results}

In comparison with a preceding investigation at the age of 4 years, MRI at the age of 9 years revealed a distinct progression of white matter abnormalities. There was slight dilation of lateral ventricles, predominantly of posterior horns, and atrophy of the vermis cerebelli. Fig. 1 (left column) demonstrates diffuse changes of both supratentorial and infratentorial white matter with almost homogenous hyperintensity on T2-weighted images (Fig. 1A,C) and an inhomogeneous, moderately low signal in T1-weighted images (Fig. 1E,G). U-fibers and corpus callosum were spared. A follow-up MRI study at the age of 12 years (Fig. 1, right column) showed a more pronounced dilation of internal and external CSF spaces as well as a further reduction of unaffected white matter. Especially lesions of cerebellar white matter had markedly increased (Fig. 1G,H). Although the images have been chosen to properly indicate the locations selected for MRS and therefore are suboptimal for demonstration of enlargement of ventricles, progressive widening of external CSF spaces is recognizable in temporo-parietal cortex (Fig. 1D,F) and cerebellum (Fig. 1H).

Fig. 2 depicts MR spectra from four different brain regions obtained at the age of 9 and 12 years. Visual inspection reveals reduced tNAA as well as elevated Cho in white matter and cerebellum. Pertinent findings confirm the MRI observation of predominant disturbances in cerebral and cerebellar white matter. The quantitative analysis of absolute metabolite concentrations in Table 1 provides a more detailed picture of metabolic alterations. In particular, at the age of 9 years, cerebral white matter from both hemispheres showed a marked reduction of tNAA $(-44 \%)$ accompanied by increased concentrations of Cho $(+56 \%)$, Ins $(+100 \%)$, and Lac. Similar though even more pronounced alterations were found in cerebellum with an additional elevation of $\mathrm{tCr}$ $(+17 \%)$. Also cortical gray matter, which appeared normal on MRI, presented with metabolic alterations. Whereas the gray matter level of tNAA was normal, the concentrations of Cho $(+73 \%)$, Ins $(+79 \%)$, and $\operatorname{tCr}(+17 \%)$ were enhanced. When comparing these results with those of the follow-up 
examination, the latter exhibited less increased concentrations of Cho and Lac but otherwise a similarly abnormal metabolic pattern.

\section{Discussion}

The MRI findings in our patient with GAN are in line with the features reported in the literature, for example, in cortical and cerebellar white matter of a 21-year-old woman with CNS symptoms including seizures but only mild symptoms of peripheral neuropathy [7]. To the best of our knowledge, the present proton MRS study is the first attempt to characterize metabolic alterations in the brain of a patient with GAN and CNS involvement.

As a main finding the reduction of tNAA in white matter and cerebellum suggests a damage or even loss of neuroaxonal tissue. The very strong elevation of Cho and Ins at the age of 9 years probably reflects both glial proliferation and active demyelination. Glial proliferation may be predominantly ascribed to astrocytes because of the pronounced increase of Ins. The assumption of active demyelination subsequent to axonal damage is also supported by the detection of Lac, which most likely indicates the anaerobic metabolism of infiltrating macrophages removing cellular decomposition products during acute phases of the disease.

Cerebral white matter at the age of 12 years showed unchanged low tNAA, normalized Lac, and slightly less increased Cho and Ins. These metabolic disturbances, consistent with fading of active demyelination, hint to a regional stabilization. Similarly, elevations of Cho and Ins in cerebellum are less pronounced than at 9 years, but the ongoing atrophy as detected by MRI and the strong increase of Lac point to progressive cellular disruption in cerebellum. Accordingly tNAA concentration remains close to detectability. These findings are in line with clinical progression of the disease, dominated by almost total loss of all major motor functions, progressive ataxia and brainstem dysfunction as well as clear signs of dementia. The metabolic characterization of cortical gray matter is in line with neuronal preservation (normal tNAA) and glial proliferation (increased Cho and Ins). Together these effects result in a higher cell density, which leads to elevated $\mathrm{tCr}$ values, because of its even distribution amongst neuronal and glial cells.

The metabolic pattern of strong disturbances in white matter and comparatively mild alterations in gray matter are in line with the clinical picture of a white matter disease dominated by spasticity and ataxia interfering with the longstanding signs of peripheral neuropathy. Only late in the course symptoms of dementia became apparent. Clinical as well as MRI and MRS follow-up demonstrated a cranio-caudal progress of the disease with increasing dysfunction of cerebellum and brainstem.

The metabolic abnormalities obtained in vivo are in excellent agreement with histopathological findings reported previously. Postmortem studies of CNS involvement in GAN have been performed in several patients [10]. The detailed neuropathological report by Thomas et al. of a patient who died at the age of 18 years described a normal brain weight, slight dilation of ventricles, and thinning of corpus callosum [11]. White matter of centrum semiovale was grayish discolored and showed additional spongy changes in cerebellum. Subcortical arcuate fibers were macroscopically well preserved. There was a distal axonopathy most severely affecting the corticospinal tracts, middle cerebellar peduncles, and posterior columns, as well as olivocerebellar degeneration. In line with the MRS findings of our patient presented here, histological examination of cerebral cortex revealed increased numbers of astrocytes and scattered Rosenthal fibers. The cerebral white matter was severely gliotic containing only few giant axons. Optic nerves and tracts showed fiber loss and gliosis. Giant axons and Rosenthal fibers were found in pons and medulla. The cerebellar white matter was much depleted of nerve fibers and consisted mainly of astrocytic processes and Rosenthal fibers. Kretzschmar et al. reported similar histopathological features [10] on the original case of Berg et al. [4], and emphasized the presence of Rosenthal fibers in CNS as a conspicuous and probably regular finding in typical cases of GAN.

In summary, this proton MRS study of GAN provides an in vivo assessment of metabolic alterations in various regions of the brain that reflect histopathological changes such as neuroaxonal damage or loss, active demyelination, glial proliferation, and enhanced cellular density in close agreement with the results of postmortem analyses. In a more general perspective, the disturbed metabolite pattern found in white matter resembles the typical abnormalities observed in leukodystrophies with active demyelination, e.g. metachromatic leukodystrophy [21] and Krabbe's disease [16].

\section{Acknowledgements}

P.J.W.P. was supported by a fellowship of the European Community (ERBCH-BGCT 940722).

\section{References}

[1] Prineas JW, Ouvrier RA, Wright RG, Walsh JC, McLeod JG. Giant axonal neuropathy - a generalized disorder of cytoplasmic microfilament formation. J Neuropathol Exp Neurol 1976;35:458-470.

[2] Pena SD. Giant axonal neuropathy: an inborn error of organization of intermediate filaments. Muscle Nerve 1982;5:166-172.

[3] Ouvrier RA. Giant axonal neuropathy. A review. Brain Dev 1989;11:207-214.

[4] Berg BO, Rosenberg SH, Asbury AK. Giant axonal neuropathy. Pediatrics 1972;49:894-899.

[5] Carpenter S, Karpati G, Andermann F, Gold R. Giant axonal neuropathy. A clinically and morphologically distinct neurological disease. Arch Neurol 1974;31:312-316.

[6] Igisu H, Ohta M, Tabira T, Hosokawa S, Goto I, Kuroiwa Y. Giant 
axonal neuropathy. A clinical entity affecting the central as well as the peripheral nervous system. Neurology 1975;25:717-721.

[7] Lampl Y, Eshel Y, Ben-David E, Gilad R, Sarova-Pinhas I, Sandbank U. Giant axonal neuropathy with predominant central nervous system manifestations. Dev Med Child Neurol 1992;34:164-169.

[8] Richen P, Tandan R. Giant axonal neuropathy: Progressive clinical and radiologic CNS involvement. Neurology 1992;42:2220-2222.

[9] Majnemer A, Rosenblatt B, Watters G, Andermann F. Giant axonal neuropathy: central abnormalities demonstrated by evoked potentials. Ann Neurol 1986;19:394-396.

[10] Kretzschmar HA, Berg BO, Davis RL. Giant axonal neuropathy. A neuropathological study. Acta Neuropathol (Berl) 1987;73:138-144.

[11] Thomas C, Love S, Powell HC, Schultz P, Lampert PW. Giant axonal neuropathy: correlation of clinical findings with postmortem neuropathology. Ann Neurol 1987;22:79-84.

[12] Flanigan KM, Crawford TO, Griffin JW, Goebel HH, Kohlschütter A, Ranells J, et al. Localization of the giant axonal neuropathy gene to chromosome 16q24. Ann Neurol 1998;43:143-148.

[13] Bomont P, Cavalier L, Blondeau F, Ben Hamida C, Belal S, Tazir M, et al. The gene encoding gigaxonin, a new member of the cytoskeletal $\mathrm{BTB} /$ kelch repeat family, is mutated in giant axonal neuropathy. Nat Genet 2000;26:370-374.

[14] Treiber-Held S, Budjarjo-Welim H, Riemann D, Richter J, Kretzschmar HA, Hanefeld F. Giant axonal neuropathy: a generalized disorder of intermediate filaments with longitudinal grooves in the hair. Neuropediatrics 1994;25:89-93.
[15] Frahm J, Michaelis T, Merboldt KD, Bruhn H, Gyngell ML, Hänicke W. Improvements in localized proton NMR spectroscopy of human brain. Water suppression, short echo times, and $1 \mathrm{ml}$ resolution. J Magn Reson 1990;90:464-473.

[16] Frahm J, Hanefeld F. Localized proton magnetic resonance spectroscopy of brain disorders in childhood. In: Bachelard HS, editor. Magnetic resonance spectroscopy and imaging in neurochemistry, New York: Plenum, 1997. pp. 329-402.

[17] Pouwels PJ, Brockmann K, Kruse B, Wilken B, Wick M, Hanefeld F, et al. Regional age dependence of human brain metabolites from infancy to adulthood as detected by quantitative localized proton MRS. Pediatr Res 1999;46:474-485.

[18] Birken DL, Oldendorf WH. N-acetyl-L-aspartic acid: a literature review of a compound prominent in $1^{\mathrm{H}}$ NMR spectroscopic studies of the brain. Neurosci Biobehav Rev 1989;13:23-31.

[19] Brand A, Richter-Landsberg C, Leibfritz D. Multinuclear NMR studies on the energy metabolism of glial and neuronal cells. Dev Neurosci 1993;15:289-298.

[20] Pouwels P, Hanefeld F, Frahm J. Proton MRS in Pelizaeus-Merzbacher disease. Neuropediatrics 1997;28:355-356.

[21] Kruse B, Hanefeld F, Christen HJ, Bruhn H, Michaelis T, Hänicke W, et al. Alterations of brain metabolites in metachromatic leukodystrophy as detected by localized proton magnetic resonance spectroscopy in vivo. J Neurol 1993;241:68-74. 